Draft version April 19, 2022

Preprint typeset using LATEX style emulateapj v. 11/10/09

\title{
MULTI-LAYER STUDY OF WAVE PROPAGATION IN SUNSPOTS
}

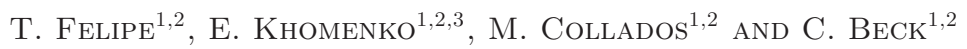 \\ Draft version April 19, 2022
}

\begin{abstract}
We analyze the propagation of waves in sunspots from the photosphere to the chromosphere using time series of co-spatial Ca II H intensity spectra (including its line blends) and polarimetric spectra of Si I $\lambda 10827$ and the He I $\lambda 10830$ multiplet. From the Doppler shifts of these lines we retrieve the variation of the velocity along the line-of-sight at several heights. Phase spectra are used to obtain the relation between the oscillatory signals. Our analysis reveals standing waves at frequencies lower than $4 \mathrm{mHz}$ and a continuous propagation of waves at higher frequencies, which steepen into shocks in the chromosphere when approaching the formation height of the Ca II H core. The observed nonlinearities are weaker in Ca II H than in He I lines. Our analysis suggests that the Ca II H core forms at a lower height than the He I $\lambda 10830$ line: a time delay of about $20 \mathrm{~s}$ is measured between the Doppler signal detected at both wavelengths. We fit a model of linear slow magnetoacoustic wave propagation in a stratified atmosphere with radiative losses according to Newton's cooling law to the phase spectra and derive the difference in the formation height of the spectral lines. We show that the linear model describes well the wave propagation up to the formation height of Ca II H, where non-linearities start to become very important.
\end{abstract}

Subject headings: Sun: magnetic fields; Sun: oscillations

\section{INTRODUCTION}

Since the first detection of waves in sunspots (Beckers \& Tallant 1969), many studies have been carried out to understand the physics of these waves, from the observational as well as theoretical point of view. Waves in sunspots are different from those observed in quiet Sun due to the presence of the magnetic field. They show a variety of behavior depending on the height and the region of the sunspot where they are observed. Usually, these waves are classified as photospheric umbral oscillations, chromospheric umbral oscillations, and running penumbral waves (Lites 1992). However, all these kind of waves seem to be different manifestations of the same global propagation of magneto-acoustic waves (Rouppe van der Voort et al. 2003). The reasons to study waves extend beyond the derivation of their properties, because waves can also be used as independent diagnostic of sunspot structure (Duvall et al. 1996).

At the photosphere in the umbra, the power spectra of the oscillations are quite similar to the corresponding ones of the quiet Sun, with a broad distribution of frequencies and a clear peak at about $3 \mathrm{mHz}$ (Thomas et al. 1982). In sunspots, these oscillations in the $5 \mathrm{~min}$ band have reduced amplitudes compared to the surrounding quiet photosphere (Abdelatif et al. 1986). The magnetic field also modifies the $p$-modes and produces new modes of fluctuations not present in the quiet Sun (Cally \& Bogdan 1997; Khomenko et al. 2009). Generally, most of the photospheric umbra is covered by coherent oscillations (Kobanov 1990). Waves are usually studied by measuring the fluctuations of intensity and line-of-sight velocity from Doppler shift, but they are also

\footnotetext{
${ }^{1}$ Instituto de Astrofísica de Canarias, 38205, C/ Vía Láctea, s/n, La Laguna, Tenerife, Spain

2 Departamento de Astrofísica, Universidad de La Laguna, 38205, La Laguna, Tenerife, Spain

${ }^{3}$ Main Astronomical Observatory, NAS, 03680, Kyiv, Ukraine
}

supposed to affect magnetic field. From full Stokes inversions, Lites et al. (1998) found an upper limit of $4 \mathrm{G}$ for the amplitude of 5 minute oscillations in magnetic field strength, while Bellot Rubio et al. (2000) detected variations around 7-11 G. In a later work, Khomenko et al. (2003) interpreted magnetic field oscillations as being due to fast and slow magneto-hydrodynamic (MHD) wave modes, producing both intrinsic variations of the magnetic field and those due to the shift of the line forming region.

The chromosphere of sunspots is dominated by $3 \mathrm{~min}$ oscillations. The power spectra usually show sharp peaks around $5-6 \mathrm{mHz}$, and the power drops gradually to noise values in the interval from $8 \mathrm{mHz}$ to $10-15 \mathrm{mHz}$ (Lites 1984). Several spectral lines, formed at different heights from the photosphere to the chromosphere, can be used to sample the wave propagation properties. Simultaneous time-series of these spectral lines are a powerful tool for studying sunspot waves. From phase spectra between the umbral oscillations observed in the photospheric line Fe I $\lambda 5233$ and in $\mathrm{H} \alpha$, Giovanelli et al. (1978) found that the phase delay indicates upward wave propagation. Lites (1984) inferred that slow mode waves propagate upward into the chromosphere in the frequency band around $6.5 \mathrm{mHz}$, based on the phase differences between the oscillations of CaII $\lambda$ 8498, Ca II $\lambda 8542$ and Fe I $\lambda$ 5434. From the study of the Doppler shifts observed in the intensity profiles of the He I $\lambda 10830$ multiplet, Lites (1986) presented evidence of shock formation at the chromosphere. Centeno et al. (2006) reproduced the phase spectra between chromospheric and photospheric velocity oscillations with a model of linear vertical propagation of slow magnetoacoustic waves in a stratified magnetized atmosphere that accounts for radiative loses, finding a good agreement between the theoretically computed time delay, and the one obtained from the cross-correlation of photospheric Si I $\lambda 10827$ and chromospheric He I $\lambda 10830$ 
velocity maps, filtered around the $6 \mathrm{mHz}$ band. They showed that the chromospheric $6 \mathrm{mHz}$ signal is a result of linear wave propagation of the photospheric perturbations in the $6 \mathrm{mHz}$ range, rather than the consequence of the nonlinear interaction of photospheric modes as proposed by Gurman \& Leibacher (1984).

The works cited above were limited to the study of oscillations at only two heights (one photospheric and one chromospheric), separated by several hundreds of kilometers. It is thus interesting to perform a more detailed sampling of the sunspot atmosphere, using more spectral lines formed at several intermediate heights between these two regions. On the one hand, observationally detected spatial wave patterns in sunspots are rather complex (Bogdan \& Judge 2006). On the other hand, recent numerical simulations of waves in sunspots also suggest a complex picture of the fast and slow magnetoacoustic waves propagating simultaneously in the same layers but in different directions and with different phase speeds (Khomenko \& Collados 2009). This requires a more refined multi-layer study of sunspot waves. Studies of this kind often represent an observational challenge since several spectral lines have to be detected simultaneously not only in intensity but also in polarized light. In our paper, we report on such multi-line spectropolarimetric observations. Our aim is to cover the gap between the photospheric and chromospheric signals and analyze sunspot oscillations at the formation heights of several spectral lines formed between Si I and He I. For that we use simultaneous observations obtained with two instruments, the POlarimetric LIttrow Spectrograph (POLIS, Beck et al. 2005b) and the Tenerife Infrared Polarimeter II (TIP-II, Collados et al. 2007), attached to the German Vacuum Tower telescope at the Observatorio del Teide at Tenerife. Apart from information about waves, our multi-layer study has also allowed us to estimate the formation heights of the spectral lines in the sunspot atmosphere, including $\mathrm{Ca}$ II $\mathrm{H}$ line, several Fe I blends in the wing of this line and the infrared lines of Si I at $\lambda$ $10827 \AA$ and He I at $\lambda 10830 \AA$.

The structure of the paper is the following. In Sect. 2 the observations and data reduction are explained. Section 3 describes the analysis of the velocity oscillations at several heights. The results are discussed in Sect. 4. which also presents our conclusions.

\section{OBSERVATION AND DATA REDUCTION}

The observations analyzed in this work were obtained on 2007 August 28 with two different instruments, POLIS and TIP-II, attached to the German Vacuum Tower Telescope (VTT) at the Observatorio del Teide. Simultaneous and co-spatial scans of a sunspot located near the center of the Sun $(\mu=0.96)$ were taken with both instruments. The slit was placed over the center of the sunspot. The observations were obtained with real-time seeing correction by the Kiepenheuer-Institute adaptive optics system (von der Luehe et al. 2003).

The spectra of the blue channel of POLIS include Ca II H $\lambda 3968 \AA$ intensity profiles and some photospheric line blends in the wings of $\mathrm{CaII} \mathrm{H}$, covering a spectral range from $3964.9 \AA$ to $3971.3 \AA$ with a spectral sampling of $20 \mathrm{~m} \AA$ pixel $^{-1}$ and a spatial sampling of $0^{\prime \prime} 29$ per pixel. The Ca spectra were reduced for the flat-

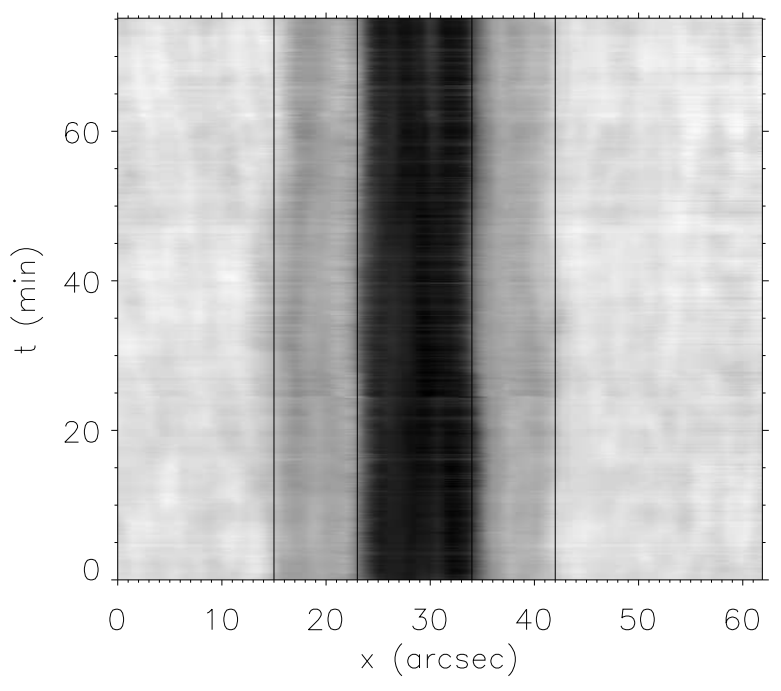

FIG. 1.- Intensity of IR continuum. Vertical lines mark the position of quiet Sun-penumbra and penumbra-umbra boundaries. Time increases from bottom to top.

field (Beck et al. 2005a, b) , and were also corrected for the transmission curve of the order-selecting interference filter in front of the camera. For the wavelength calibration, the line-core positions of the iron lines at 3965.45, $3966.07,3966.63,3967.42$ and $3969.26 \AA$ in an average quiet Sun region were determined by a second order polynomial fit. We then determined the wavelength scale that matched best all the position values.

TIP-II yielded the four Stokes parameters $I Q U V$ in a spectral range from $10822.7 \AA$ to $10833.7 \AA$ with a spectral sampling of $11 \mathrm{~m} \AA$ pixel $^{-1}$ and a spatial sampling of $0^{\prime \prime} 18$ per pixel. This spectral region contains information about two different heights of the solar atmosphere due to the presence of two spectral lines. The Si I line at $10827.09 \AA$ is formed at photospheric heights, whereas the He I $\lambda 10830$ triplet, which includes a weak blue component at $10829.09 \AA$ and two blended red components at 10830.25 and $10830.34 \AA$, forms in the chromosphere (Centeno et al. 2008). In this case, the wavelength calibration was done using the Si I and He I lines as references.

The full data set consists of three temporal series; each of them lasting about one hour. Three scan steps with $0^{\prime \prime} 5$ step width were taken for the two first series, while in the last series only two spatial positions were used. The cadence was different for all series. Table 1 shows the time step between two spectra taken at the same spatial position, the time when the observation were obtained and the number of repetitions of each series.

TABLE 1

SUMMARY OF OBSERVATIONS:

\begin{tabular}{ccccc}
\hline & $\mathrm{t}_{\text {start }}(\mathrm{UT})$ & $\mathrm{t}_{\text {end }}(\mathrm{UT})$ & $\Delta \mathrm{t}(\mathrm{s})$ & $\mathrm{N}$ spectra \\
\hline Series 1 & $07: 55: 02$ & $08: 54: 48$ & 21 & 170 \\
Series 2 & $09: 01: 02$ & $10: 15: 25$ & 18 & 250 \\
Series 3 & $10: 43: 34$ & $11: 58: 43$ & 7.5 & 600 \\
\hline
\end{tabular}

Due to the differential refraction in the earth atmo- 

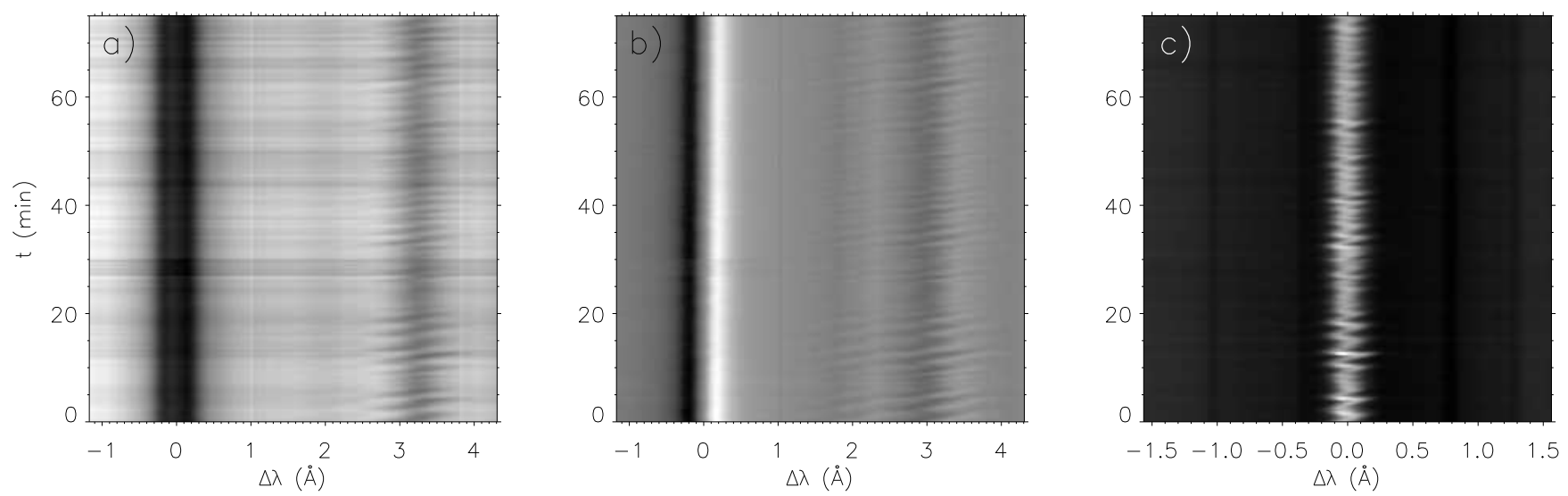

FIG. 2.- Temporal evolution at one position in the umbral region of series \#2. Left: Si I and He I intensity; center: Si I and He I Stokes $V$; right: Ca II H intensity. The horizontal axis represents wavelength, with the origin at the position of the Si I $\lambda 10827 \AA$ rest wavelength in (a) and (b) and at the position of the Ca II H $\lambda 3968 \AA$ rest wavelength in (c). The vertical axis represents time, increasing from bottom to top.

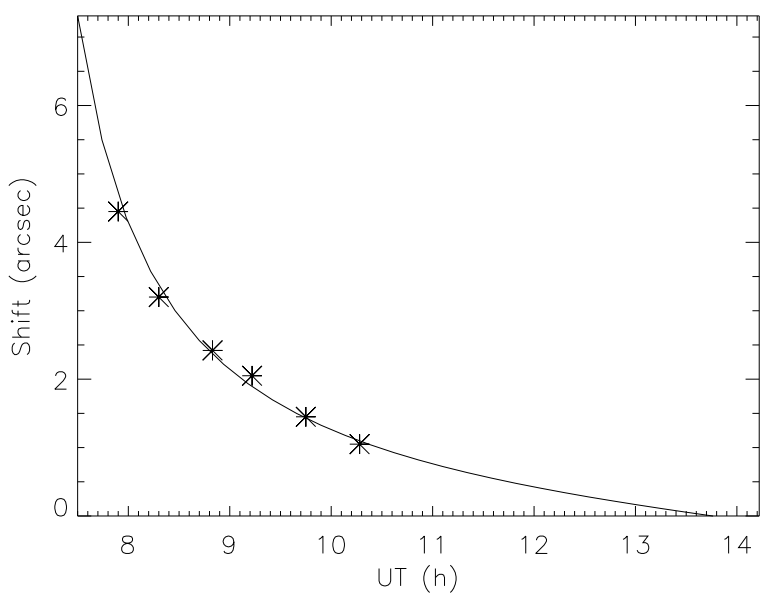

FIG. 3.- Temporal variation of the theoretical spatial displacement perpendicular to the slit due to differential refraction (Solid line). Asterisks mark the values measured at the telescope.

sphere (e.g., Filippenko 1982), the spectra of POLIS and TIP-II are not fully co-spatial. The spatial displacement of the two wavelengths (3968 $\AA, 10830 \AA$ ) perpendicular to the slit depends on the date and time of the observations, the slit orientation, and the location of the first coelostat mirror (see Appendix A of Beck et al. 2008). On the first day of the observation campaign, we took a set of large-area scans at different times for an accurate determination of the displacement. The solid line in Fig. 3 shows the theoretically predicted spatial displacement perpendicular to the slit due to differential refraction, while the asterisks are the measured displacements; the match between both is remarkable. To guarantee an overlap between the observations in the two wavelengths, we thus positioned the scan mirror inside of POLIS at the beginning of each observation such that it compensated the spatial displacement for about the middle of the observation. Moreover, small repeated scans of 2-3 slit spatial positions, separated by $0^{\prime \prime} 5$, were taken in order to sample a wider region of the Sun and prevent possible errors between the theoretical differential refraction and the actual one.

Figure 1 shows an intensity map from a wavelength in the infrared continuum. The regions of quiet Sun, penumbra, and umbra of the sunspot are well defined, the vertical lines indicate the boundaries between these areas. The temporal evolution of the Stokes $I$ and Stokes $V$ spectra for the TIP data and the intensity around the Ca II $\mathrm{H}$ core in the POLIS data are plotted in Fig. 2 at a fixed position inside the umbra. The Stokes $I$ and $V$ profiles from TIP contain the Si I line at $\Delta \lambda=0 \AA$ (the rest wavelength of the silicon line was determined from the quiet Sun region and was set as the origin) and the He I line at $\Delta \lambda=3.25 \AA$. The helium line profile shows periodic shifts with large displacements to the blue and the red. The core of the CaII H line shows a strong emission peak inside the umbra. The Doppler shift of this emission peak develop a saw-tooth pattern, similar to the He I line (see also Rouppe van der Voort et al. 2003).

\section{DATA ANALYSIS AND RESULTS}

In this paper, we focus on the line-of-sight (LOS) velocities. For all the spectral lines besides CaII H, Doppler velocities were inferred by measuring the position of the intensity minimum. The wavelengths close to the core of the line were fitted with a second order polynomial. The location of the minimum of the parabola was taken as the line-core position. This procedure was performed to obtain the Doppler shifts of Si I, He I, and the Fe I lines at $3965.45,3966.07,3966.63,3967.42$ and $3969.26 \AA$. In the case of the TIP data, the Doppler shift of the Stokes $V$ zero crossing, where the polarization signal intersects the zero level, was derived as well. The Doppler shifts from the intensity and Stokes $V$ profiles are very similar due to the large magnetic filling factor in the sunspot umbra.

The behavior of the Ca II $\mathrm{H}$ core is different. It exhibits a prominent peak at the center of the line in highly magnetized regions (top line of Fig. 4), while in a field-free atmosphere the center of the line has a minimum between two lobes at both sides with their corresponding maxima (bottom line of Fig. 4) (see also, e.g., Liu \& Smith 1972). In the umbra, the Doppler shift was retrieved from the spectral displacement of the maximum of the core emission. In the low magnetized, region it was obtained from the shift of the central minimum. All in all, we have obtained maps of the LOS velocity at each spatial point 


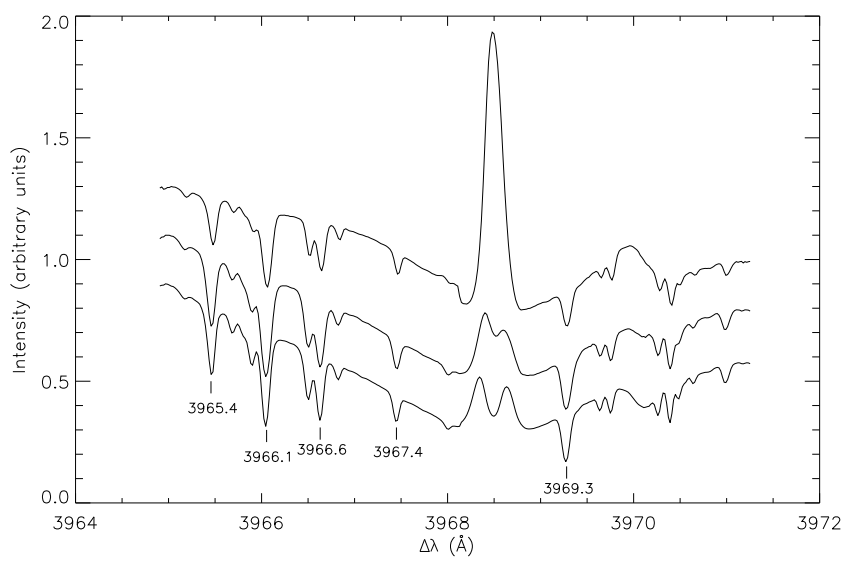

FIG. 4.- Averaged intensity profile of the Ca II $\mathrm{H}$ line at different locations. From bottom to top: quiet Sun, penumbra, and umbra. The intensity scale is different for all of them. The profiles have been displaced from each other. The Fe I lines analyzed in this work are marked.

covered by the slit at the formation heights of the eight spectral lines, except for HeI, whose line depth in the non-magnetized regions is too small to determine its location.

We have selected the scan steps which give the best alignment of the data from both instruments to obtain a better correction of the differential refraction. The use of different steps introduce a systematic delay between the oscillatory signals from POLIS and TIP-II, since the same spatial location is observed with a time lag. However, in the case of the data from series 3, the theoretical correction of the differential refraction worked perfectly and the optimal alignment was found using the simultaneous scan steps. This data set has been chosen to calculate the phase difference spectra from Section 3.3 . An additional finer alignment along the slit between TIP and POLIS was done by a cross-correlation of the velocity maps of two spectral lines formed at similar heights, using the line pair Ca II $\mathrm{H}$ and $\mathrm{He}$ I for the chromosphere, and Si I and Fe I $\lambda 3969.26 \AA$ for the photosphere. Finally, we resampled all velocity maps with the sampling of the POLIS Ca II H data, i.e., 0"29 per pixel.

\subsection{LOS velocity}

Figure 5 shows the temporal evolution of the LOS velocities in the sunspot region obtained from the Doppler shift of several spectral lines, sorted by formation height. Negative velocities (appearing as black shaded regions) indicate upflows, where matter is approaching the observer, while white regions are downflows. Figures 5(a-d) reveal a similar pattern since all these lines are formed at photospheric heights. The velocity maps of the Fe I line blends of $\mathrm{Ca}$ II $\mathrm{H}$ have lower quality than the Si I maps, especially inside the umbra, due to the coarser spectral sampling and the small line depth of the lines.

The velocity field at chromospheric heights is given in Fig. 5e ( $\mathrm{Ca}$ II H) and 5f (He I). Both figures show a similar wave pattern and similar amplitudes, but, as a more detailed analysis will reveal, the velocity oscillations of the $\mathrm{He}$ I line are slightly retarded relative to those in $\mathrm{Ca}$. The wave pattern measured at the chromosphere in the umbral region has a smaller spatial coherence than that observed in the photosphere. It also differs in its larger peak-to-peak variations of about $8 \mathrm{~km} \mathrm{~s}^{-1}$ and its period of about three minutes.

In the region of the penumbra, which can be seen in Fig. 5f at a position between $19^{\prime \prime}$ and $23^{\prime \prime}$ and between $34^{\prime \prime}$ and $39^{\prime \prime}$, there is also a characteristic pattern of alternating positive and negative velocities, but with lower amplitudes than the waves in the umbra and with longer periods, corresponding to running penumbral waves (see, e.g., Giovanelli 1972; Zirin \& Stein 1972; Bloomfield et al. 2007). They start at the inner penumbra, and their wave front is delayed in the regions of the outer penumbra, so their propagation through the penumbra appears as a slope in the diagram. An example of such a slope can be seen in an area between $19^{\prime \prime}$ and $23^{\prime \prime}$ during the first 22 minutes (white rectangle). This slope is smaller near the outer penumbra, indicating a decrease of the propagation speed as the disturbance travels from the inner to the outer penumbral boundary. The estimation of the velocity for the $\mathrm{Ca}$ II $\mathrm{H}$ line core (Fig. 5f) in the penumbral region is poorer due to the change of the shape of the line that does not have a prominent emission peak any more (see middle curve in Fig. 4).

The left panels of Fig. 6] show the histograms of the LOS velocities in the umbra of the sunspot obtained from all the spectral lines used in this study. We fitted a Gaussian to the distributions to estimate the root-meansquare (rms) velocity value. The histograms are sorted from bottom to top with increasing rms velocity, with Si I having the smallest and He I the largest rms velocity. The Fe I lines at 3966.0 and $3966.6 \AA$ have an identical rms velocity value, as they are formed at close heights. This is also suggested by their nearly identical line depth (see Fig. 4). From these histograms and the variation of the rms velocities, we obtain the first estimate of the relative formation height of the spectral lines since we expect a monotonic increase of the rms velocity with height.

For comparison, the right panels of Fig. [ show the histograms of LOS velocities for the same lines in a region of the quiet Sun. The He I histogram is not shown because the He I absorption in quiet Sun is too low to retrieve a velocity. The quiet Sun histograms also show the increase of the rms velocity with the formation height, although the order of the rms velocity slightly differs from the one obtained in the sunspot, since all the Fe I lines form in a thin layer. As the oscillatory power in sunspots is suppressed (see, for example, Hindman et al. 1997), the rms velocities in the quiet Sun are larger. Another possible source of the broadening of the quiet Sun velocity histogram can come from the granulation velocities not present inside the umbra. We did not remove the granular component from the velocity variations in the quiet Sun and it can contribute to some extent to the overall rms velocity variations (see, e.g. Kostyk \& Khomenko 2002).

Figure 7 shows an example of the temporal evolution of the LOS velocity obtained from the chromospheric He I line and the CaII H line core, the four photospheric Fe I lines, and the photospheric Si I line at one location inside the umbra of the sunspot, indicated in all panels of Fig. 5 with a vertical dashed line at $x=28^{\prime \prime}$. The plots are sorted from bottom to top with increasing formation height, as retrieved from Fig. 6. A comparison between 

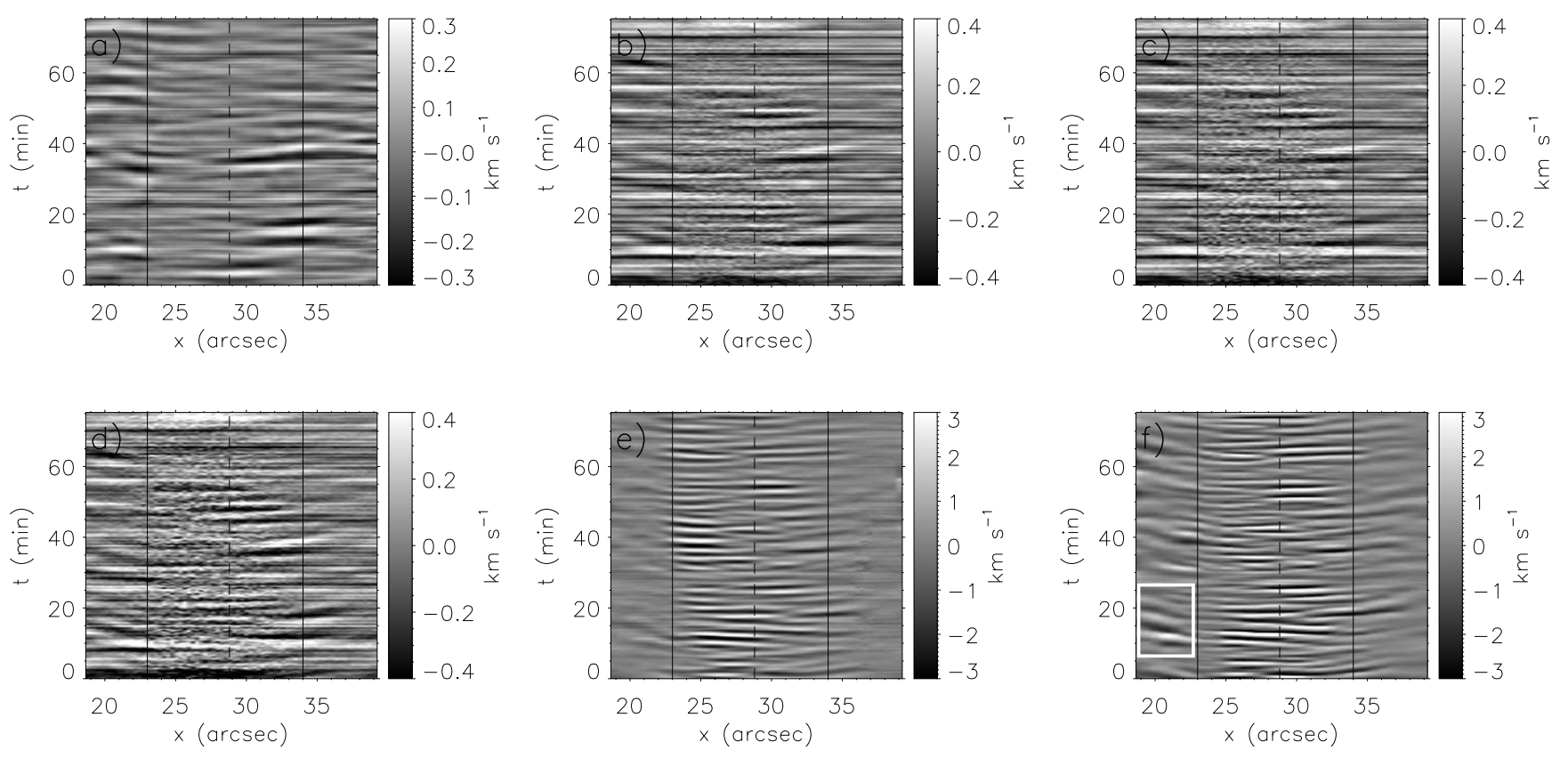

FIG. 5.- Velocity maps in the sunspot during series \#2 for the different spectral lines. The horizontal axis represents the position along the slit and the vertical axis represents time. Black color indicates negative velocities (upflow), white color positive velocities (downflow). Top row, left to right: Si I $\lambda 10827$ (a), Fe I $\lambda$ 3966.1 (b), Fe I $\lambda 3966.6$ (c). Bottom row, left to right: Fe I $\lambda 3969.3$ (d), Ca II H 3968.5 (e), He I $\lambda 10830$ (f). Vertical solid lines represents the limits of the umbra. The vertical dashed line denotes the location of the spectra shown in Fig. 7 The white rectangle in (f) marks an area of running penumbral waves.

the bottom and top panels (which show the Si I and He I velocities, respectively) reveals the differences in period and amplitude of the waves at photospheric and chromospheric heights. Between these two layers, the rest of the spectral lines sample different heights of the atmosphere. At photospheric heights (panels c-g), the pattern of waves is similar and the signals of the lines with higher formation heights are slightly delayed (see e.g. the peak at $t=49$ min marked with a dashed line). We can see that higher layers have larger amplitudes (note that the top two panels have a different scale for the velocity). The temporal evolution of the LOS velocity of the two chromospheric lines (panels a-b) is almost identical, but the amplitudes are higher in the case of the He I line. For instance from minute 10 to 25 , it is clearly seen that the oscillations measured with the He I line and $\mathrm{Ca}$ II $\mathrm{H}$ core have a saw-tooth profile that indicates the presence of a shock wave train, with a slow increase of the velocity followed by a sudden decrease. There is a phase difference of about $20 \mathrm{~s}$ between the $\mathrm{Ca}$ II $\mathrm{H}$ line core and the He I line velocities, in the sense that the oscillatory signal reaches the formation height of the $\mathrm{Ca}$ II $\mathrm{H}$ line core $20 \mathrm{~s}$ before that of the He I line.

Table 2 lists the rms velocities in the quiet Sun (except HeI) and in the sunspot for all the spectral lines, together with the ratios between the quiet Sun and the sunspot velocities. The relative increase of the rms velocity of the quiet Sun to the rms velocity of the sunspot decreases with the height in the atmosphere, from 2.80 at the photospheric height of the formation of Si I to 1.29 at the chromospheric height of the formation of $\mathrm{Ca}$ II $\mathrm{H}$ line core. The rate of the increase of the rms velocity in the sunspot is faster, and the difference between velocities from lines formed at higher layers is smaller. The growth of the amplitude of the oscillations with height is scaled by the pressure scale height, $H_{0}=R T / \mu g$, where $T$ is the temperature, $R$ is the gas constant, $g$ is the gravity and $\mu$ is the mean weight of the atoms. In the umbra the temperature is lower and $H_{0}$ is smaller than in the quiet Sun atmosphere, so the amplitude of umbral oscillations rises faster.

\subsection{Power Spectra}

Figure 8 shows the normalized average power spectra of LOS velocity of the two chromospheric (He I and Ca II H) and two of the photospheric (Si I and Fe I $\lambda$ 3969.3) lines inside the umbra of the sunspot. We chose this iron line because it has better signal to noise and its formation height is distant from the layer where Si I is formed. In the photosphere (bottom panel), the power is concentrated between 2 and $4 \mathrm{mHz}$, corresponding to the 5 minute band, with a maximum peak at $3.5 \mathrm{mHz}$. Both spectral lines peak at the same frequency, although the power of the Fe I line is slightly higher. The increase of the power at frequencies above $4.5 \mathrm{mHz}$ is more impor-

TABLE 2

RMS VELOCITIES IN MS ${ }^{-1}$ IN THE SUNSPOt AND THE QUIET SUn, AND THEIR RATIO (3RD COLUMN).

\begin{tabular}{cccc} 
line & umbra & QS & ratio QS/umbra \\
\hline Si I & 90 & 250 & 2.80 \\
Fe I 3965.4 & 170 & 256 & 1.51 \\
Fe I 3966.1 & 174 & 275 & 1.58 \\
Fe I 3966.6 & 180 & 273 & 1.52 \\
Fe I 3969.3 & 201 & 306 & 1.53 \\
Ca II H & 510 & 659 & 1.29 \\
He I & 726 & - & - \\
\hline
\end{tabular}



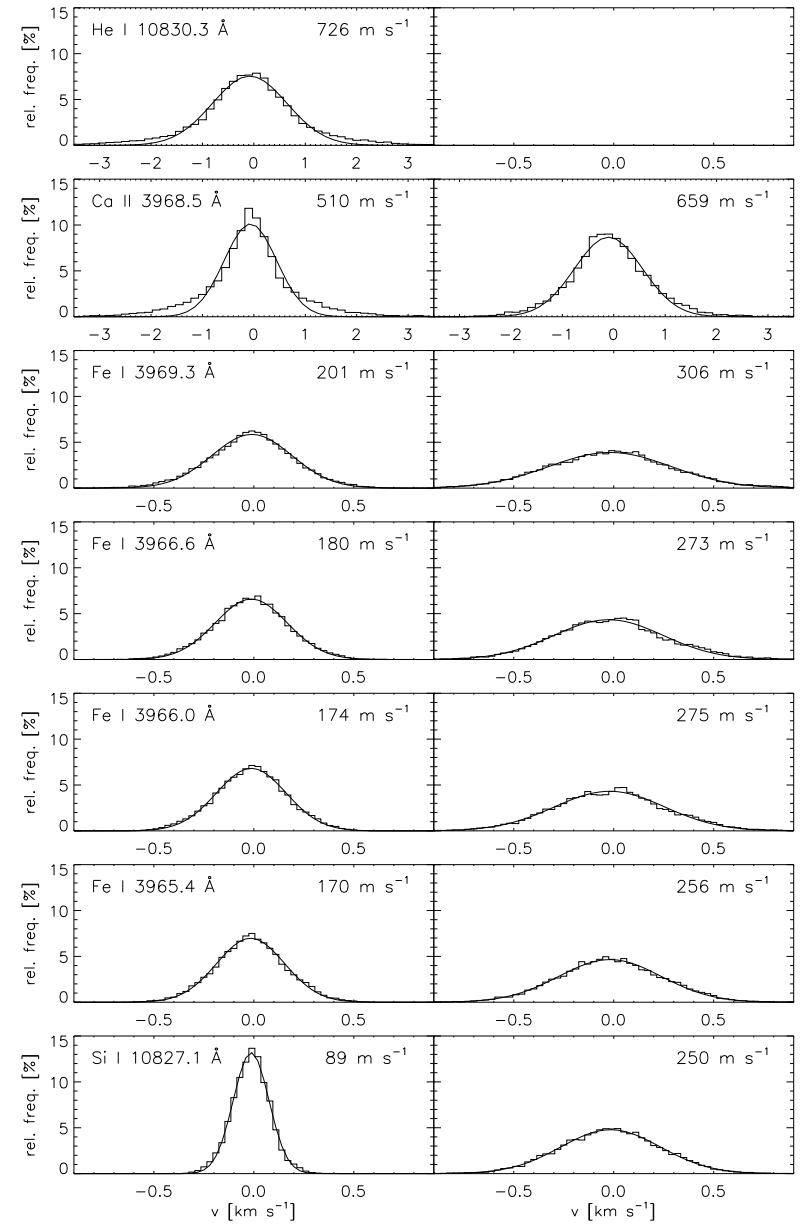

FIG. 6. - Histograms of LOS velocity measured with several spectral lines. The left column corresponds to the umbra, the right column to the quiet Sun. From bottom to top: Si I, Fe I $\lambda 3965.4$ Fe I $\lambda$ 3966.1, Fe I $\lambda$ 3966.6, Fe I $\lambda$ 3969.3, Ca II H and He I. Solid lines represent the Gaussian fit; its width is indicated in each plot.

tant than the one for frequencies below this value.

The velocity power spectra of both chromospheric lines (top panel) have a broad distribution of frequencies, with the largest power being in the band from 5 to $10 \mathrm{mHz}$. The chromospheric power spectrum has a maximum at $6.2 \mathrm{mHz}$ and several secondary peaks around it (see for comparison Lites 1986). These frequency peaks correspond to the chromospheric 3 minutes oscillations. At the highest peak of the power spectra, both Ca II H and He I have almost the same power, but for those frequencies with lower power, the power of the He I is increased comparing to the Ca II H. Note that at heights sampled by our spectral lines we do not find a continuous transition from the peak at $3.5 \mathrm{mHz}$ to the one at $6.2 \mathrm{mHz}$, but rather a discontinuous behavior between the photospheric and chromospheric power spectra. However, the prominent secondary peak around $5.5 \mathrm{mHz}$ in the power spectra of the Fe I line is much more obvious than the corresponding in the Si I power spectra, which could indicate some transition towards higher frequencies in the power spectra as the waves propagate upward from the formation height of the Si I line to the Fe I lines.

\subsection{Phase spectra}

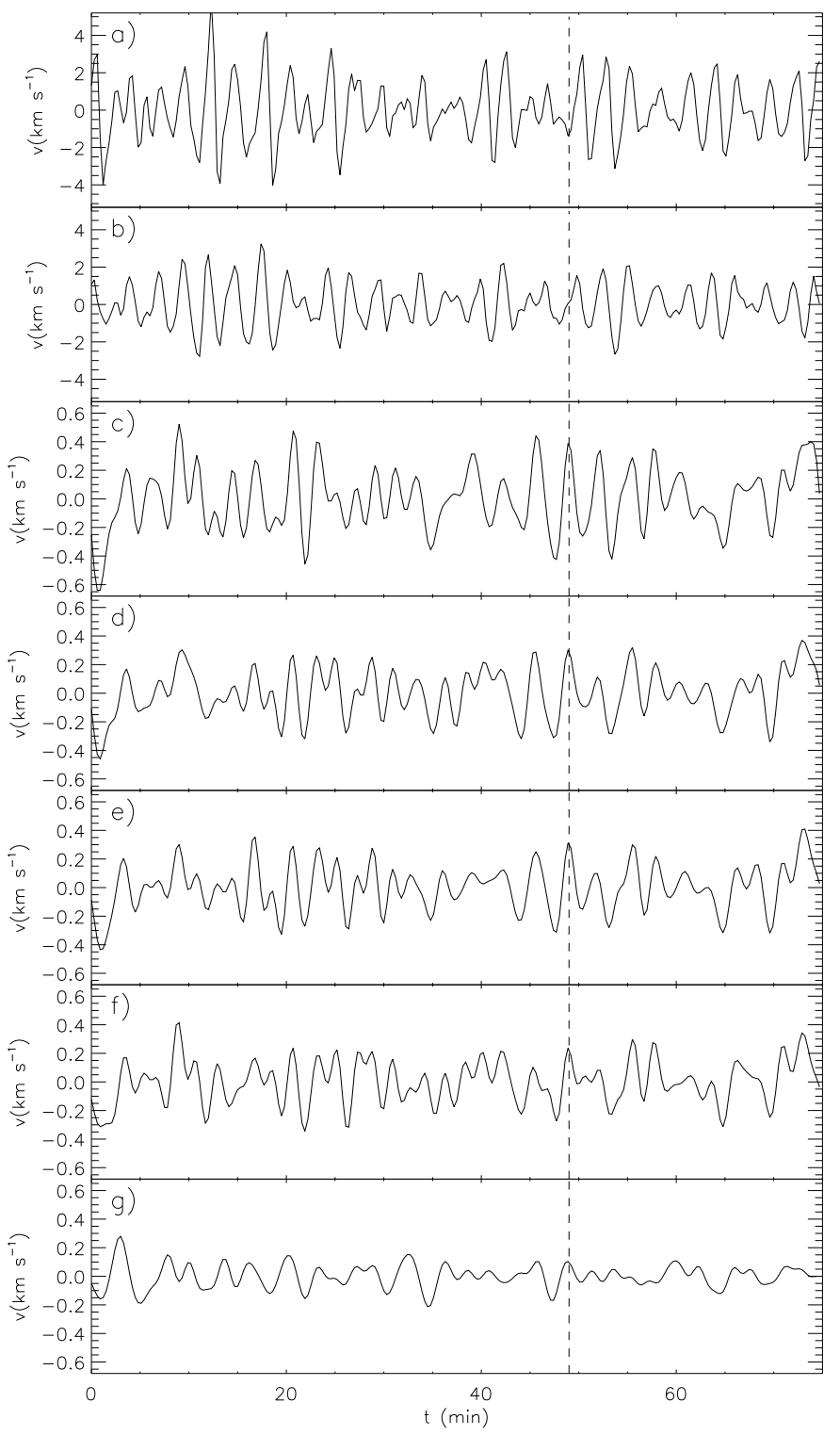

FIG. 7.- Velocity at a fixed position in the umbra for different spectral lines, sorted by formation height: He I $\lambda 10830$ (a), Ca II H 3968.5 (b), Fe I $\lambda 3969.3$ (c), Fe I $\lambda 3966.6$ (d), Fe I $\lambda 3966.1$ (e), Fe I $\lambda 3965.4$ (f), Si I $\lambda 10827.1(\mathrm{~g})$. The vertical dashed line at $t=49$ min marks a prominent velocity peak.

A phase diagram gives the phase difference $(\Delta \phi)$ between two signals. In our study, we use $\Delta \phi$ to measure the time delay between the oscillatory velocity signals from two spectral lines and assume that the difference between them is mainly due to the difference of the formation height of the two lines. In the following, we will show the phase difference spectra between different combinations of pairs of spectral lines used in this work. To obtain the phase spectra, we treated each spatial point separately and calculated the Fourier-transform of the temporal evolution of the respective velocities. We derived the phases, and from them the phase difference of the two signals as a function of the frequency. There is a $2 \pi$ ambiguity in the computation of the phase value, so all phase differences have been projected in the range $\pm \pi$. Then we calculated histograms of the relative oc- 


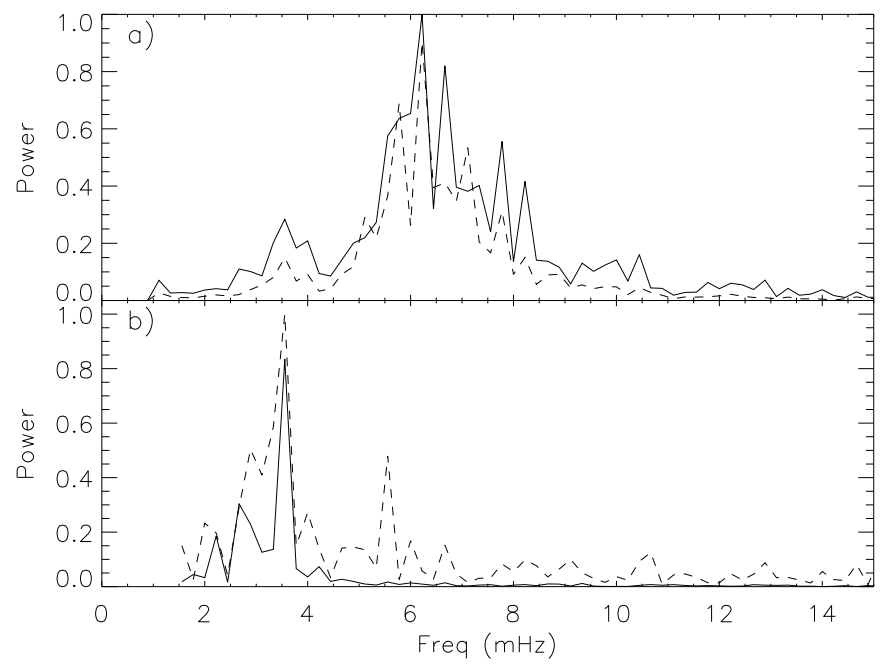

FIG. 8.- Average umbral power spectra of the LOS velocities. Top: chromospheric lines, He I (solid line) and CaII H (dashed line), both are normalized to the maximum power of the He I line; bottom: photospheric lines, Si I line (solid line) and Fe I $3969.3 \AA$ (dashed line), both are normalized to the maximum power of the Fe I line.

currence of a given value of the phase differences at each frequency taking into account all the corresponding spatial points (see also Krijger et al. 2001, and references therein). We obtained the data displayed in Figs. 9 [15.

In addition to the phase difference spectra, we calculated the coherence spectra. They provide an estimate of the statistical validity of the phase and power spectra. Considering $n$ pairs of signals $x_{k}(t)$ and $y_{k}(t)$, whose Fourier transforms are $\bar{X}_{k}(\omega)$ and $\bar{Y}_{k}(\omega)$, respectively, the coherence is defined as

$$
P_{x y}(\omega)=\frac{\frac{1}{n}\left|\sum_{k}^{n}\right| \bar{X}_{k}(\omega)|| \bar{Y}_{k}(\omega) \mid e^{i \Delta \phi_{k}(\omega) \mid}}{\frac{1}{n} \sum_{k}^{n} \sqrt{\left|\bar{X}_{k}(\omega)\right|^{2}\left|\bar{Y}_{k}(\omega)\right|^{2}}}
$$

where $\Delta \phi_{k}(\omega)=\phi_{x k}(\omega)-\phi_{y k}(\omega)$. In our case, the subindex $k$ covers the spatial position. The coherence evaluates statistically for every frequency $\omega$ the relation of the $\Delta \phi_{k}(\omega)$ for the $n k$-signals. It takes the value 1 when $\Delta \phi_{k}(\omega)$ is the same for all the $k$. If the phase difference of the different $k$ is arbitrary, the coherence takes very low values. We selected a confidence limit at 0.7 , and for frequencies with coherence above this value we consider the phase spectra to be reliable.

We also analyzed the increase of the amplitude of the oscillations. We calculated the amplification spectra as the ratio between the power at two layers, both of them averaged all over the umbra:

$$
A_{x y}=\frac{\sum_{k}^{n}\left|\bar{Y}_{k}(\omega)\right|^{2}}{\sum_{k}^{n}\left|\bar{X}_{k}(\omega)\right|^{2}} .
$$

\subsubsection{Theoretical model}

Following Centeno et al. (2006), the observations were compared with a model of linear vertical propagation of slow magneto-acoustic wave in an isothermal atmosphere that includes radiative losses described by Newton's cooling law. Assuming that the amplitude of the vertical velocity changes with height by

$$
V(z)=V_{0} e^{z /\left(2 H_{0}\right)} e^{i k_{z} z}
$$

the dispersion relation for such waves is

$$
k_{z}^{2}=\frac{\omega^{2}-\hat{\omega}_{a c}^{2}}{\hat{c}^{2}},
$$

where

$$
\hat{\omega}_{a c}=\hat{c} / 2 H_{0}, \hat{c}^{2}=\hat{\gamma} g H_{0}, \hat{\gamma}=\frac{1-\gamma i \omega \tau_{R}}{1-i \omega \tau_{R}}
$$

and $\tau_{R}$ is the radiative cooling time for an optically thin perturbation (Spiegel 1957):

$$
\tau_{R}=\rho c_{v} /\left(16 \chi \sigma_{R} T^{3}\right) .
$$

The phase difference between oscillations at two heights is calculated as the difference of the imaginary part of the argument of the complex exponential in Eq. 3. that is, $\Delta \phi=k_{R} \Delta z$, where $\Delta z$ is the geometric distance between the two heights and $k_{R}$ is the real part of $k_{z}$. The amplification spectrum is given by the ratio between the amplitude at the two layers and is obtained as $A=e^{2\left(1 /\left(2 H_{0}\right)-k_{I}\right) \Delta z}$, with $k_{I}$ being the imaginary part of $k_{z}$.

This model allows us to fit the phase and amplification spectra with three free parameters: the temperature of the atmosphere, $T$, the difference in height between two lines, $\Delta z$, and the typical time scale in which the temperature fluctuations are damped radiatively, $\tau_{R}$. These free parameters are manually tuned to match the effective cut-off frequency and the slope (including its variations) of the phase difference spectra in the regime of propagating waves above the cut-off frequency.

\subsubsection{Si $\mathrm{I}-H e \mathrm{I}$ phase spectra}

Figure 9 shows the phase difference between the velocity signals measured in the photospheric Si I line and the chromospheric He I line. The phase difference is zero for frequencies below $4 \mathrm{mHz}$. At these frequencies the coherence is high, except in the range between 1 and 2.5 $\mathrm{mHz}$, and the atmosphere oscillates as a whole, i.e., the waves are stationary. From $4 \mathrm{mHz}$ to $7 \mathrm{mHz}$, the phase difference increases linearly with the frequency and the coherence is (more or less) above the confidence limit. It indicates that waves at these frequencies propagate from the photospheric layer, where the Si I line forms, to the chromospheric layer, where He I forms. The phase difference spectra for higher frequencies are very noisy, and no meaningful conclusions are possible.

The bottom panel of Fig. 9 shows the ratio of chromospheric to photospheric power as a function of frequency. We fit both the phase difference and the amplification spectra simultaneously. The parameters of the fit are listed in Table 3. The solid red line in the phase diagram in Fig. (9) represents the phase difference according to the model presented in Sect. 3.3.1 calculated with the parameters that best fit the observations. The theoretical amplification spectra matches rather well to the observational one, with an order of magnitude agreement in the amplification factor. 

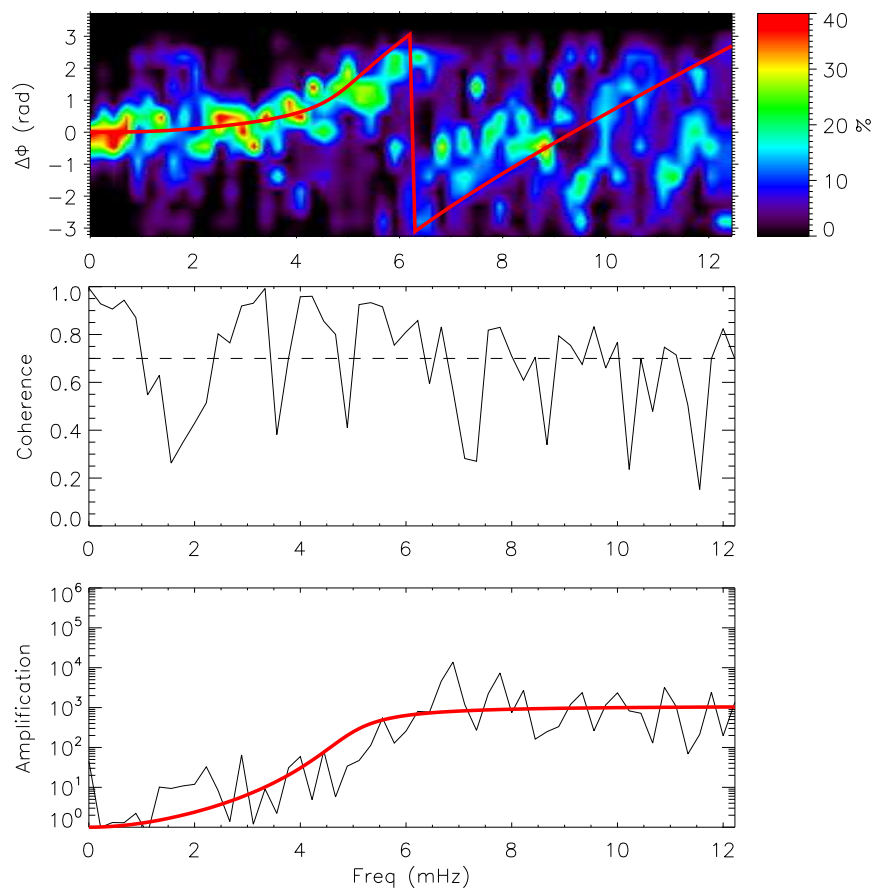

FIG. 9.- Top: Phase spectra between the LOS velocities of the Si I and the HeI lines. The color code shows the relative occurrence of a given phase shift. The red line represents the best fit from the theoretical model. Middle: Coherence spectra. The horizontal dashed line at 0.7 marks the confidence limit. Bottom: Amplification spectra. The red line represents the best fit from the theoretical model.

TABLE 3

Best-Fit PARAMETERS OF THE THEORETICAL MODEL

\begin{tabular}{cccc}
\hline Line pair & $\mathrm{T}(\mathrm{K})$ & $\Delta z(\mathrm{~km})$ & $\tau_{R}(\mathrm{~s})$ \\
\hline Si-He & 4500 & 900 & 45 \\
Si-Ca & 4000 & 650 & 45 \\
Fe 3969.3-He & 4000 & 450 & 45 \\
Fe 3969.3-Si & 4000 & -280 & 30 \\
Fe 3969.3-Si (quiet sun) & 4500 & -200 & 30 \\
Fe 3965.4-Fe 3969.3 & 3500 & 30 & 45 \\
Fe 3966.0-Fe 3969.3 & 3500 & 20 & 45 \\
Fe 3965.4-Fe 3966.0 & 3500 & 10 & 45 \\
Ca-He & 6000 & 100 & 15 \\
\hline
\end{tabular}

\subsubsection{Si $\mathrm{I}-\mathrm{Ca}$ II $H$ phase spectra}

Figure 10 shows the phase, coherence and amplification spectra between the velocity measured with the Si I line and the core of $\mathrm{CaII} \mathrm{H}$ line. Frequencies below 1 $\mathrm{mHz}$ have a very low coherence, so these values are not reliable. Surprisingly, frequencies in the range between 1 and $2.5 \mathrm{mHz}$ present an anomalous behavior, with a phase difference around $2.5 \mathrm{rad}$ and a high amplification, while their coherence is remarkably high. The long period of waves with these frequencies hinders their analysis, so further observations with longer temporal series are required to study this behavior. The rest of the phase spectra is similar to the one between Si I and He I, with zero phase difference between 2.5 and $4 \mathrm{mHz}$, indicating stationary waves, and an almost linear increase of the phase difference between 4 and $7 \mathrm{mHz}$, corresponding to upwards propagating waves. In all this frequency range, the coherence is high. However, in this case the slope of
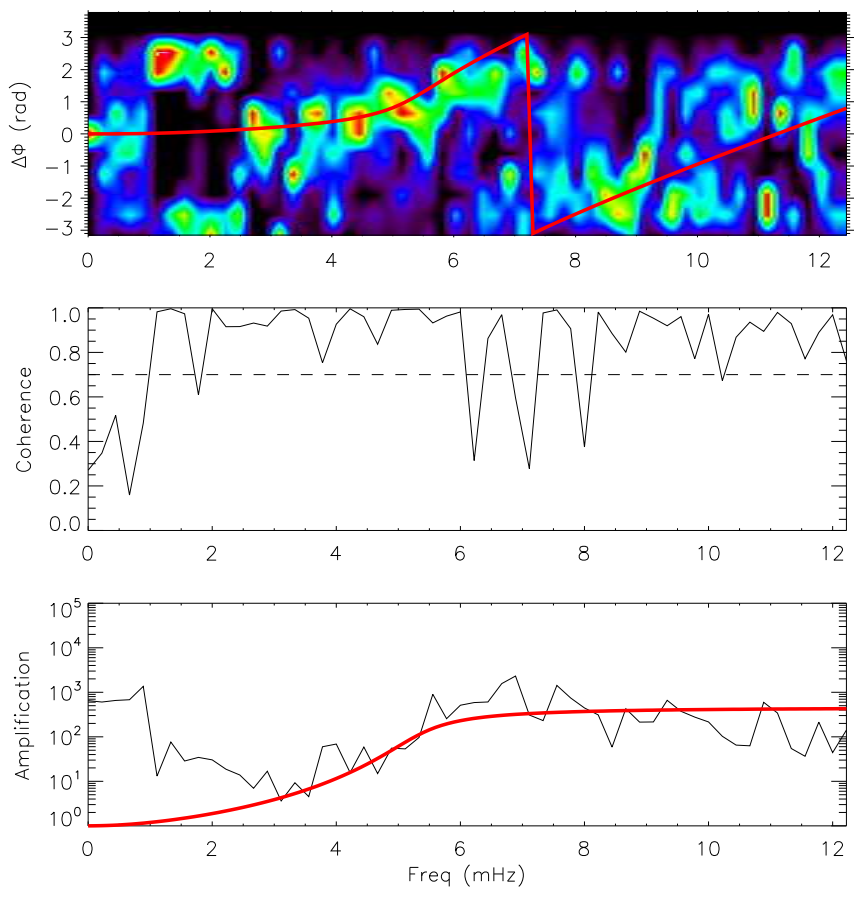

FIG. 10.- Phase, coherence and amplification spectra between Si I and CaII H. The format of the figure is the same as Fig. 9]

the increase of the phase difference is smaller than in the previous one, and the amplification also has lower values. It means that the phase delay between the velocities at the formation height of these two lines is smaller than the delay between Si I and He I and the amplitude of the waves at the formation height of He I is larger than that of waves at the layer where Ca II H forms. Both the phase and amplification spectra locate the formation height of the $\mathrm{Ca}$ II $\mathrm{H}$ core below the He I line.

The parameters retrieved from the fit of the phase and amplification spectra to the theoretical model are listed in Table 3. Comparing the ones retrieved for the line pair Si I-He I with the pair Si I-Ca II H, the CaII H core formation height in the umbra is around $250 \mathrm{~km}$ below the He I line. The temperature obtained for the phase difference between Si I and CaII H is smaller by $500 \mathrm{~K}$; waves traveling from the formation height of $\mathrm{Ca}$ II $\mathrm{H}$ core to that of He I pass presumably through a region where the temperature is increasing.

\subsubsection{Fe I phase spectra}

To study the properties of oscillations at photospheric heights, phase diagrams between pairs of the photospheric lines were calculated. We can assume that the Fe I lines in the $\mathrm{CaII} \mathrm{H}$ wing form at three different heights according to their line depth (Fig. 3) and to the width of the velocity histograms (Fig. 6). We take the lines Fe I $\lambda$ 3965.4, Fe I $\lambda 3966.0$ and Fe I $\lambda 3969.3$ as representative of these heights, since Fe I $\lambda 3966.6$ and Fe I $\lambda$ 3967.4 seem to form at a similar height as Fe I $\lambda$ 3966.0. As an example, Fig. 11 shows the phase spectra, coherence and amplification spectra obtained between between Fe I $\lambda 3966.0$ and Fe I $\lambda$ 3969.3. The phase spectrum shows that the phase difference is almost zero for all the frequencies that can be trusted according to the coherence spectra (from 0 to $10 \mathrm{mHz}$ ). We conclude that we can not retrieve the phase shift between the Fe I lines re- 

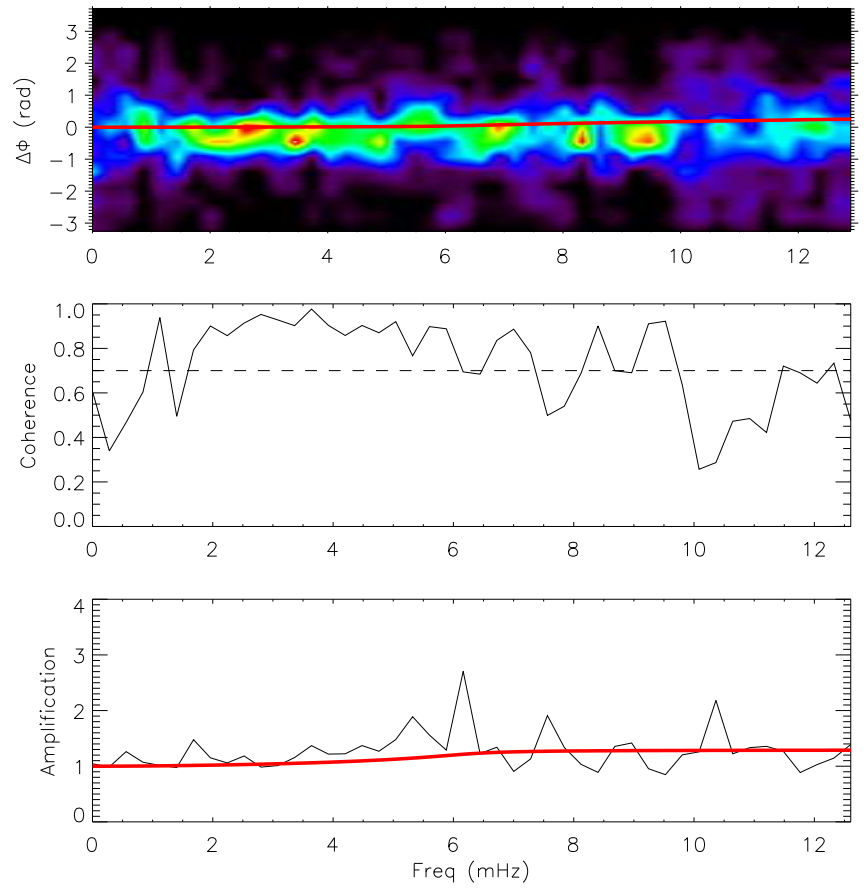

FIG. 11. - Phase, coherence and amplification spectra between FeI $\lambda 3966.0$ and Fe I $\lambda$ 3969.3. The format of the figure is the same as Fig. 9]

liably, as the geometrical difference in their heights of the formation is too small. However, the amplification spectra reflects some increase of the amplitude with height. We managed to fit both the phase and the amplification spectra with the wave model described in the previous section. These fits yield, indeed, a small geometrical height difference between the Fe I lines. The temperature and the cooling time obtained from the fit are identical for all pairs of iron lines (Table 3).

\subsubsection{Fe I-Si $\mathrm{I}$ phase spectra}

The phase difference spectrum between the FeI $\lambda$ 3969.3 line and the Si I $\lambda 10827$ line is shown in the top panel of Fig. 12. For frequencies below $2 \mathrm{mHz}$, the phase spectrum is very noisy and has no coherence (see middle panel), indicating that waves at the heights of formation of these two lines are not related. At frequencies in the band of $2.5-4 \mathrm{mHz}$, some oscillatory power is present, the coherence is high and the phase difference is close to zero, so these waves are evanescent. The phase difference spectrum for high frequencies in the range from 4 to 9 $\mathrm{mHz}$ shows a decreasing tendency, indicating that the waves reach the formation height of the Si I line before the Fe I line. This phase spectrum was also fitted with the wave propagation model. The solid red line in top and bottom panels of Fig. 12 shows the result of this fit. The amplification spectrum is not reliable at frequencies below $3 \mathrm{mHz}$ due to the low coherence, but above this value the agreement of the theoretical and observational spectra is good, indicating that the amplitude of the oscillations measured in Si I is about twice lower than the amplitude measured in the Fe I 3969.3 line.

As a summary of the propagation at photospheric heights, we conclude that all observed photospheric spectral lines (five Fe I lines and the Si l line) fluctuate with a dominant period of 5 minutes. While all the Fe I lines
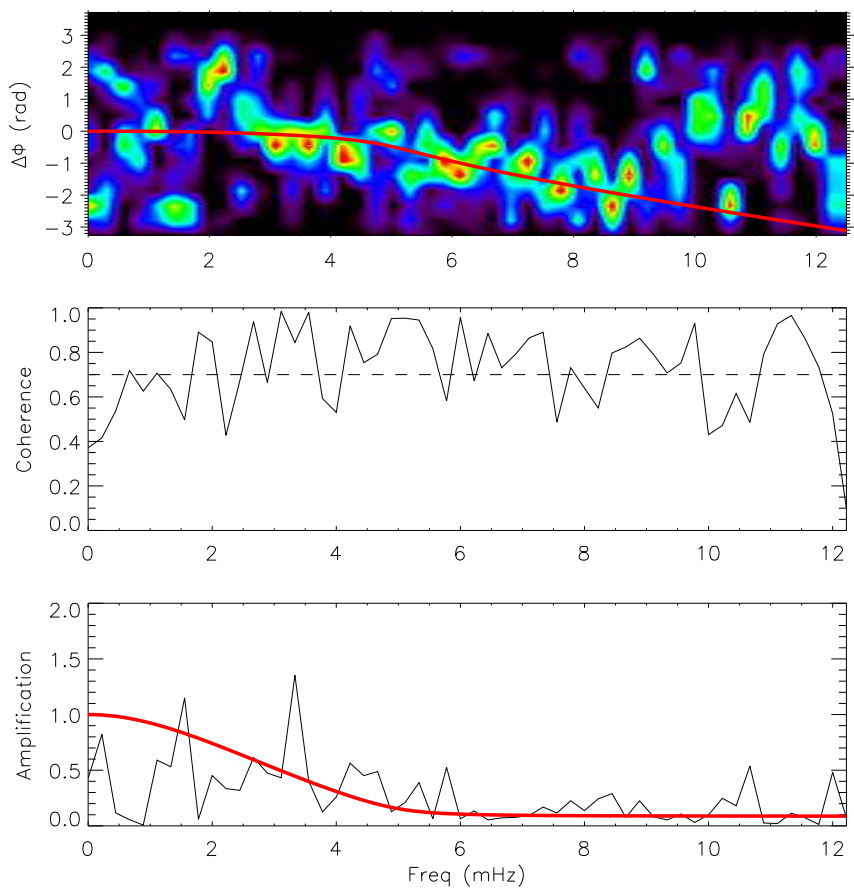

FIG. 12.- Phase, coherence and amplification spectra between Fe I 3969.3 and Si I. The format of the figure is the same as Fig. 9

have similar velocity amplitudes, with a maximum peakto-peak amplitude of around $800 \mathrm{~m} \mathrm{~s}^{-1}$, the velocity amplitude measured in Si i is clearly smaller (maximum peak-to-peak amplitude around $400 \mathrm{~m} \mathrm{~s}^{-1}$ ). According to the phase diagram between the Fe I lines (Fig. 11) and the amplification and phase diagram between a Fe I line and Si I (Fig. 12), we conclude that waves with frequencies above $4 \mathrm{mHz}$ propagate upwards at photospheric heights. They first reach the height where Si I is formed. As they propagate upward, their amplitudes increase due to the density fall-off. Then the waves reach the formation height of all the Fe I spectral lines, that all come from a thin layer and for this reason all show similar amplitudes.

\subsubsection{FeI-He I phase spectra}

According to the results extracted from the velocity statistics and the phase spectra presented before, the Fe I lines form at some height between the formation height of the Si I line and the chromospheric He I line and the CaII H core. This means that they give information about a high photospheric layer, located at an intermediate height in the propagation of the waves from the photosphere to the chromosphere. Figure 13 shows the analysis of the phase differences and amplification between the velocity signal measured at the formation height of the Fe I $\lambda 3969.3$ line and the He I line. The phase spectrum is similar to the other two between photospheric and chromospheric lines (Si I-He I and Si I-CaII H). For frequencies between 2 and $4 \mathrm{mHz}$, the phase difference is about zero, and it increases with the frequency between 4 and $7 \mathrm{mHz}$, but the slope is smaller than for Si I-He I. In the range between 2 and $7 \mathrm{mHz}$, the coherence is significant. The amplification spectrum also shows a lower amplification, compared to the Si I-He I case. The temperature, height difference and radiative damping time retrieved from the fit are listed in Table 3. 

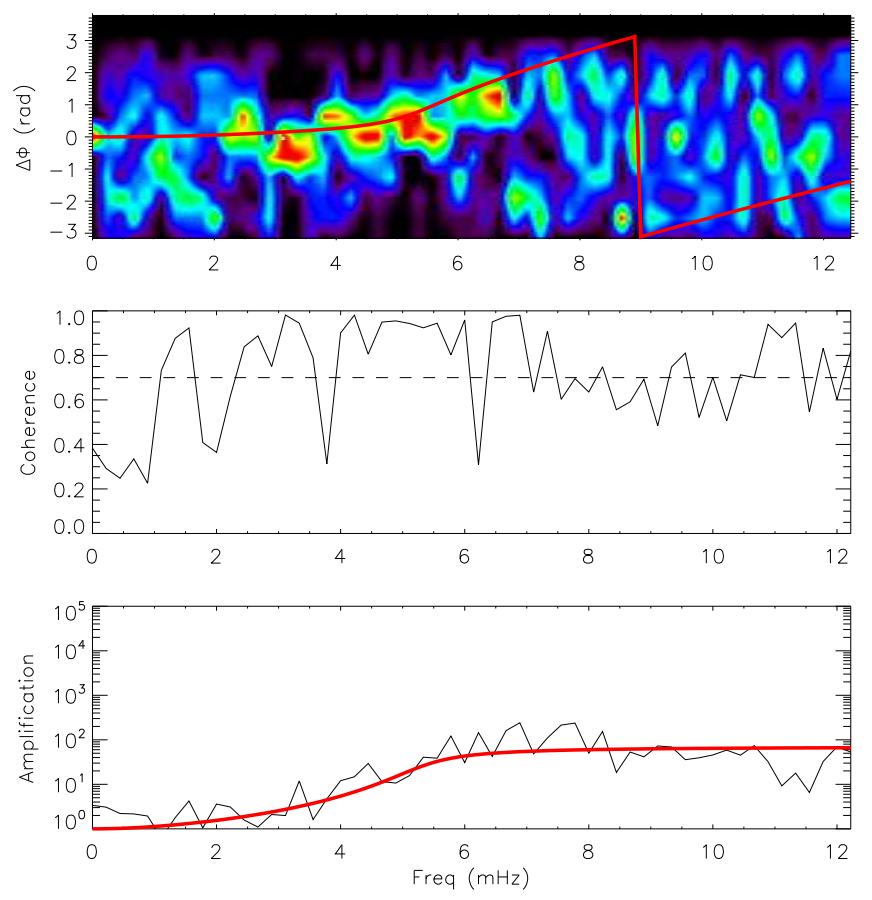

FIG. 13.- Phase, coherence and amplification spectra between Fe I $\lambda 3969.3$ and HeI. The format of the figure is the same as Fig. 9

\subsubsection{Ca II $H$-He I phase spectra}

At chromospheric heights, we have the Doppler velocities obtained from the cores of the $\mathrm{Ca}$ II $\mathrm{H}$ line and the He I $\lambda 10830$ line. The top panel of Fig. 14 shows the phase difference diagram between them. At frequencies between 1-2 $\mathrm{mHz}$, the coherence (middle panel of Fig. 14) is low and the phase spectrum is noisy. The coherence spectrum shows that the phase spectrum is reliable between 2 and $12 \mathrm{mHz}$. In the frequency band between 2 and $4 \mathrm{mHz}$, the phase difference is about 0 , indicating that there is no propagation and the waves are evanescent. From $\nu=4 \mathrm{mHz}$ to $\nu=11 \mathrm{mHz}$, the phase difference increases, starting from $\Delta \phi=0$ and showing a small positive slope. It means that as waves propagate upwards, they reach the Ca II $\mathrm{H}$ core formation height just before the He I one. The same conclusion was obtained previously from comparing the temporal variations of the Ca II H core and He I velocities presented in Fig. 77(a-b), where the oscillatory signal of He I is delayed by $20 \mathrm{~s}$ with respect to the $\mathrm{Ca}$ II $\mathrm{H}$ one.

The differences in the amplitudes between Ca II H core and He I velocities are in line with their phase spectra (top panel in Fig. 14), since the amplitude of the He I velocity is bigger than the Ca II H. A more detailed inspection of the amplification spectra between both chromospheric lines (bottom panel of Fig. 14) reveals that the oscillatory signal is amplified between 2 and $4 \mathrm{mHz}$, but there is no amplification for frequencies in the range from 4.5 to $10 \mathrm{mHz}$ (the ratio between the amplitudes is around unity). Waves in the $2-4 \mathrm{mHz}$ frequency range are evanescent, and the increase of their amplitude from the photosphere to the chromosphere is not so high, keeping them in a linear regime. At the high layers, their amplitude still increases due to the drop of the density with height. On the other hand, waves with frequencies between 4 and $10 \mathrm{mHz}$ propagate upwards to the chromo-
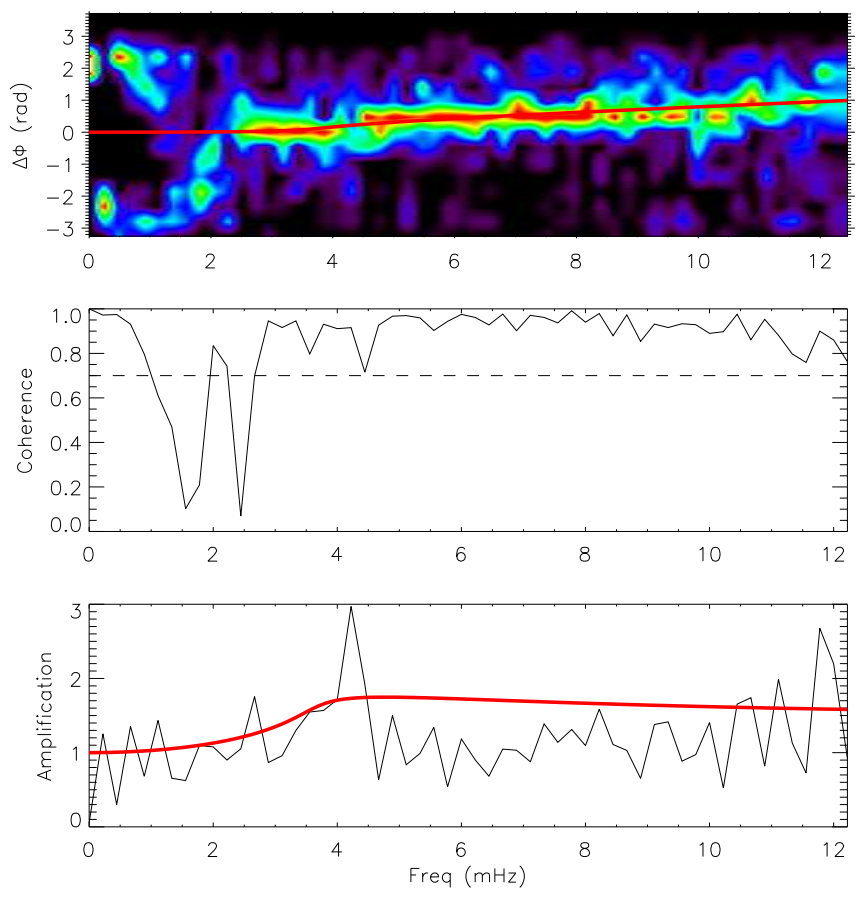

FIG. 14.- Phase, coherence and amplification spectra between Ca II H and HeI. The format of the figure is the same as Fig. 9

sphere and develop into shocks (top panels of Fig. 7). In this non-linear regime, their amplitudes do not increase with height, explaining the observational amplification spectrum around unity for frequencies in the range 4$10 \mathrm{mHz}$. The model of linear wave propagation in an isothermal stratified atmosphere predicts a higher amplification. In the case of waves with frequencies above $10 \mathrm{mHz}$, they do propagate upwards, but their amplitude at the photosphere is so low that they do not reach a non-linear regime and their amplitude still increases with height at chromospheric layers. All in all, the model is not suitable for the description of waves at heights of Ca II H core and He I formation where the non-linearities start to become important.

It is expected that the propagation of non-linear waves happens at a higher speed compared to the linear case. This would decrease the phase difference between two layers. Therefore, we can expect that the height difference between the formation layers of $\mathrm{Ca}$ II $\mathrm{H}$ and $\mathrm{He}$ I lines presented in Table 3 is underestimated by our linear model; the value of about $100 \mathrm{~km}$ is the lower limit of this difference. One of the issues that arises from this fact is the evaluation of the height range where the linear model of wave propagation is valid. It is clear that it fails between the formation height of the $\mathrm{Ca}$ II $\mathrm{H}$ core and the He I line due to the non-linearities. However, in previous sections we have applied successfully this model to the propagation between the photospheric Si I line and the chromospheric CaII H core and He I line, and between one of the Fe I lines and the He I line. It means that in most of their way from the photosphere to the chromosphere, the linear regime is a good approximation for these waves. At some layer between the formation height of the Fe I 3969.3 and the He I lines the wave propagation departs from the linear regime (cf. Carlsson \& Stein 1997). 


\subsubsection{FeI-Si I phase spectra in quiet Sun}

We calculated the phase difference spectra for a region of quiet Sun around the sunspot as well. In this region, the Stokes parameters $Q U V$ are below the level of noise, so we suppose there is no significant magnetic field. Figure 15 (top) shows the phase spectrum between the photospheric Fe I $\lambda 3969.3$ and Si I $\lambda 10827$ lines in the quiet Sun. The coherence spectrum shows high values for frequencies below $9 \mathrm{mHz}$. For frequencies below $2 \mathrm{mHz}$, the phase diagram is very noisy due to the low oscillatory power of the two velocity signals, and we do not find any clear relation between them. In the spectral range from $2 \mathrm{mHz}$ to $4 \mathrm{mHz}$, the phase difference takes a constant value around zero, while for frequencies above 4 $\mathrm{mHz}, \Delta \phi$ decreases with increasing frequency. However, the low power at the photosphere at frequencies between 6 and $8 \mathrm{mHz}$ (Fig. 8) results in a noisy phase spectrum in this band.

Comparing the phase difference diagrams between the same two lines inside the umbra of the sunspot (top panel in Fig. 12) and in the quiet Sun, we find that in quiet Sun the negative slope of the phase difference is less steep. We also fitted the quiet Sun phase difference and amplification spectra with our wave propagation model. Inside the umbra of the sunspot, the magnetic field imposes wave propagation along field lines, and thus the hypothesis of vertical propagation is justified. However, in the quiet Sun waves can propagate in different directions and this approximation may not be true. Still, we were able to find a fit matching reasonably the phase spectrum. We retrieve a lower value of formation height differences and a higher temperature in the quiet Sun compared to the umbra (see Table 3).

The bottom panel of Fig. 15 shows the amplification spectrum for this case. For frequencies in the band of 2-7 $\mathrm{mHz}$, the theoretical amplification matches the observational one.

\subsection{Comparison of the parameters of the fit with a model of sunspot}

From the fit to the phase and amplitude spectra of all the pairs of lines we obtain the temperature, the difference between the formation heights of two lines, and the cooling time that best match the observational data. Obviously, our model is simplified and has several important limitations. It only describes linear wave propagation in an isothermal stratified atmosphere, not taking into account a realistic temperature stratification of the sunspot atmosphere. In a real sunspot, the temperature varies with height, so the temperature that we obtain from the fit represents a mean value between two heights of formation. In the case of spectral lines formed at a similar height (i.e., the Fe I lines), the temperature that we find should be close to the real temperature in the layer. However, when the formation height difference $(\Delta z)$ obtained from the fit is larger (i.e., Si I-HeI), we can not assign the temperature to a certain height. The presence of shocks in the temporal evolution of the He I and Ca II H velocities (Fig. 7), together with the difficulties of the model to fit the amplification spectra between Ca II H and He I (Fig. 14), show that the propagation at these heights is non-linear. Thus our determination of the height difference between these two layers must
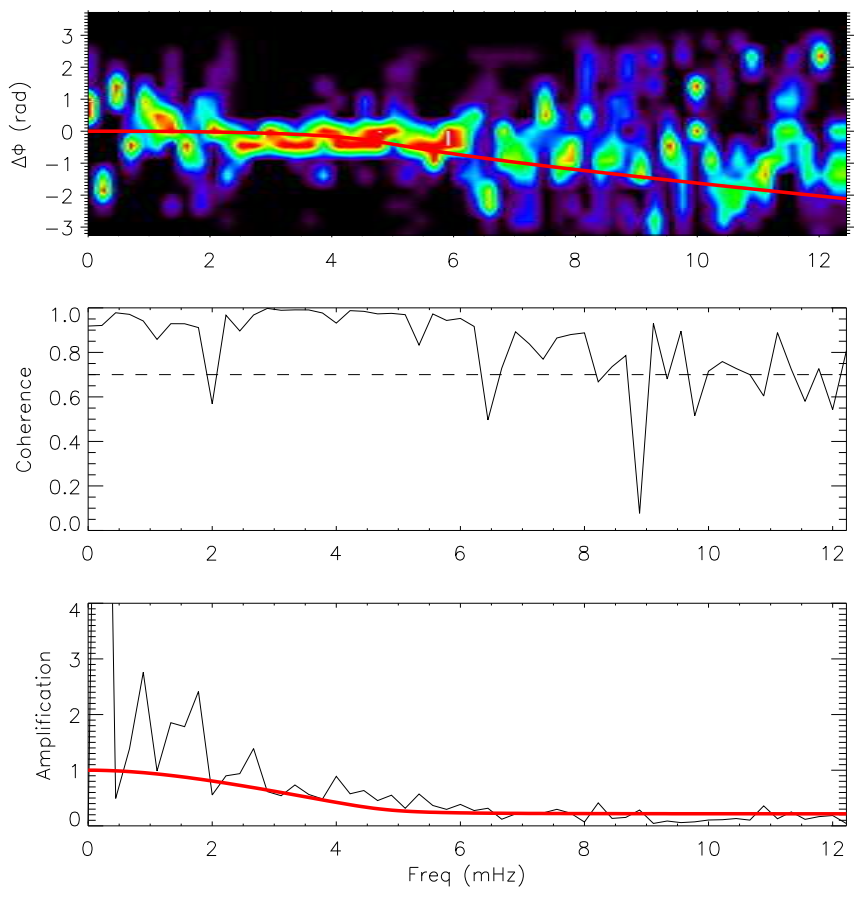

FIG. 15. - Phase, coherence and amplification spectra between Fe I $\lambda 3969.3$ and $\mathrm{Si}$ I in a quiet Sun region. The format of the figure is the same as Fig. 9

be somewhat affected by the deviations from the linear regime.

Despite these limitations, we plotted the deduced temperature values over the temperature stratification of the sunspot model of Maltby et al. (1986) (Fig. [16). The symbols in this figure mark the values retrieved from the fit to our observations. From the fit, we obtain the relative difference between the formation heights of spectral lines, not the absolute values. As reference point, we set the height of the Si I line to $\mathrm{z}=308 \mathrm{~km}$, as given by Bard \& Carlsson (2008) for the sunspot atmosphere model of Maltby et al. (1986). The formation heights of the other lines then follow from their relative distance to the Si I line (Table 3). Table 4 lists the resulting height values and the corresponding temperature.

In the case of the Fe I lines, we obtained from the low phase difference (top panel of Fig. 11) that the distances between them are very small, but the amplification spectra (bottom panel of Fig. 11) or the histograms of velocities (Fig. 6) show a certain increase of the amplitude with height. This amplification in spite of the small geometrical distance indicates a low pressure scale height $H_{0}$ and consequently a low temperature $T$. The height of the Fe I line close to the temperature minimum agrees with the results from the fit of the model to the phase and amplification spectra.

In the case of the chromospheric signals (Ca II H core and He I line), we assign to both formation heights the temperature retrieved from the fit of the phase spectra between Ca II H and He I velocities. According to the geometrical differences of Table 3 there is an uncertainty in the formation height of these two lines. On the one hand, we have set them from their $\Delta z$ with respect to the Si I line, locating the formation height of the Ca II H core at $958 \mathrm{~km}$ and the formation height of the He I line at $1208 \mathrm{~km}$. On the other hand, we have obtained an 


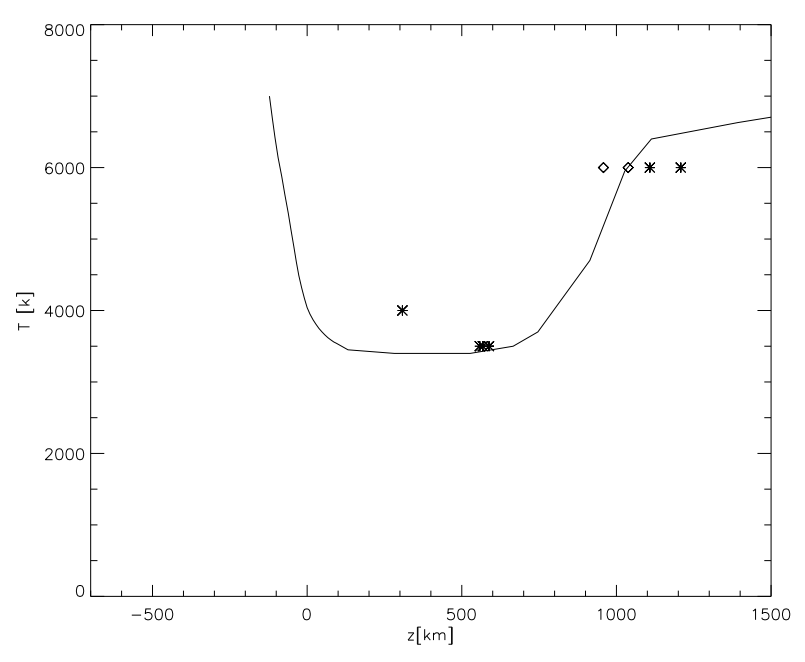

FIG. 16.- Temperature stratification in the sunspot model of Maltby et al. (1986). Asterisks represent our best-fit values to the phase difference and amplification spectra of the line pairs. Diamonds mark an alternative estimate of the formation height of the $\mathrm{Ca}$ II $\mathrm{H}$ core and the He I line.

alternative height for the $\mathrm{Ca}$ II $\mathrm{H}$ core by considering its $\Delta z$ to the He I line, and subtracting it from the previous estimate of the He I formation height. In the same way, we have located the formation height of the He I line taking into account the geometrical differences between the pairs Si I-Fe I $\lambda 3969.3$ and Fe I $\lambda$ 3969.3-HeI. Thus, we retrieved a range of heights for both chromospheric lines, as it is shown in Table 4. The height ranges we find are comparable to previous works (e.g., Lites et al.|1993; Carlsson \& Stein 1997; Centeno et al. 2009; Beck et al. 2009), even if some of these articles deal with the quiet Sun solely.

TABLE 4

FORMATION HEIGHTS AND TEMPERATURE FOR THE SPECTRAL LINES

\begin{tabular}{ccc}
\hline Spectral line & $\mathrm{z}[\mathrm{km}]$ & $\mathrm{T}[\mathrm{k}]$ \\
\hline Si I & 308 & 4000 \\
Fe I 3965.4 & 558 & 3500 \\
Fe I 3966.0 & 568 & 3500 \\
Fe I 3969.3 & 588 & 3500 \\
Ca II H & $958-1108$ & 6000 \\
He I & $1038-1208$ & 6000 \\
\hline
\end{tabular}

\section{DISCUSSION AND CONCLUSIONS}

We have presented an analysis of the LOS velocities obtained from at set of spectropolarimetric data in the near-IR spectral region around $10830 \AA$ and the optical region around $3969 \AA$ in a sunspot atmosphere and its vicinity. From these two spectral ranges, we retrieve on the one hand the Doppler velocities of the photospheric Si I $\lambda 10827$ and the chromospheric He I $\lambda 10830$ line. On the other hand, we also sample several layers between these two heights, using the Doppler shifts of the chromospheric Ca II H line core and the photospheric Fe I lines from the wings of the $\mathrm{Ca}$ II $\mathrm{H}$ line.

The histograms of LOS velocities show that the width of the velocity distribution increases with height, both in the sunspot and the quiet Sun atmosphere. Quiet Sun rms velocities are larger than those in the sunspot due to the higher power of oscillations in the quiet Sun. The growth of the amplitude of the oscillations with height is scaled by the pressure scale height $H_{0}$. It is smaller in the umbra than in the quiet Sun atmosphere, so the amplitude of umbral oscillations rises faster. This yields that the ratio of the quiet Sun and umbral rms velocities of the same lines decreases with the formation height of the lines.

The phase difference spectra of LOS velocities between several pairs of lines show upward propagating waves for frequencies higher than $4 \mathrm{mHz}$. The power at lower frequencies does not propagate up, since waves with these frequencies are evanescent. The slope of the phase spectra, together with the histograms of LOS velocity, allows us to sort all the spectral lines used in this work by their formation height.

Phase and amplification spectra were fitted to a model of linear wave propagation in a stratified atmosphere with radiative losses following the Newton cooling law. The model works reasonably well at layers below the formation height of the $\mathrm{CaII} \mathrm{H}$ line core, where waves propagate in a linear regime, while it fails in the fit of the amplification spectrum between the He I velocity and the CaII H line core velocity, due to the importance of nonlinearities at these chromospheric heights.

From the fit to the model, we retrieved the temperature, the difference in geometrical height between the formation heights of both spectral lines, and the radiative relaxation time. Setting the height of the lowermost forming line (Si I) to agree with Bard \& Carlsson (2008), the formation height of all the lines in sunspots was inferred. The Fe I lines from the wings of the Ca II H line are formed about $250 \mathrm{~km}$ above the photospheric Si I line. The relative position of the lines is well determined by the observations, since the rms velocities (Fig. 6), the power spectra (Fig. 8), and the phase and amplification spectra (Fig. 12) all indicate that the Fe I lines are formed in the upper photosphere above the Si I line. The temperatures obtained for the spectral lines then show a good agreement with the temperature stratification of the Maltby et al. (1986) sunspot model, and the formation heights are coherent with previous estimates. It must, however, be taken into account that the estimate of the formation height from response functions in Bard \& Carlsson (2008) was performed for a static atmosphere and has an strong dependence on the atmosphere model employed.

Most of the power of the photospheric lines is concentrated in a prominent peak at $3.5 \mathrm{mHz}$, in the 5 minute band. From a comparison between the power spectra of the Si I and one of the Fe I lines, it is interesting to note that the power peak is exactly at the same position, although the iron line forms at around $200 \mathrm{~km}$ above the silicon one. The same behavior was found for the chromospheric He I line and the Ca II H line core, which peak at around $6 \mathrm{mHz}$, corresponding to the 3 minute band. The maximum of the power spectra is not shifted gradually from $3.5 \mathrm{mHz}$ at the photosphere to higher frequencies at larger heights, but the photospheric and chromospheric group of lines show a discontinuous behavior. Waves at frequencies above the cut-off increase their amplitude with height faster than evanescent waves below the cut-off, resulting in larger power of 3-minute waves 
at chromospheric heights. Yet, as follows from Fig. 8b, the LOS velocity power at frequencies above $4 \mathrm{mHz}$ measured from of the Fe I line is higher than the one from the Si I line; it means that already in the upper photosphere the high-frequency power becomes important. This finding suggests that high-frequency waves prominent in the chromosphere have to be generated in the photosphere or below and their dominance at the chromospheric height is the result of their large amplitude increase. These results are consistent with those obtained from observations by Centeno et al. (2006) using two He I and Si I lines, and from numerical simulations by Felipe et al. (2010).

From the compatibility between our observations and a simple wave model, we conclude that we observe a continuous field-aligned propagation of slow magneto-acoustic waves in the upper atmosphere of sunspot. These waves first reach the formation height of Si I, then the formation height of the $\mathrm{Fe}$ I lines from the $\mathrm{Ca}$ II $\mathrm{H}$ line wing located in the upper photosphere, then the formation height of the Ca II H line core and finally that of the He I line. The propagation becomes non-linear at heights between the formation of $\mathrm{Fe}$ I lines and Ca II H line core.

This research has been funded by the Spanish MICINN through projects AYA2007-63881 and AYA2007-66502.

\section{REFERENCES}

Abdelatif, T. E., Lites, B. W., \& Thomas, J. H. 1986, ApJ, 311, 1015

Bard, S. \& Carlsson, M. 2008, ApJ, 682, 1376

Beck, C., Khomenko, E., Rezaei, R., \& Collados, M. 2009, A\&A, 507,453

Beck, C., Schlichenmaier, R., Collados, M., Bellot Rubio, L., \& Kentischer, T. 2005a, A\&A, 443, 1047

Beck, C., Schmidt, W., Kentischer, T., \& Elmore, D. 2005b, A\&A, 437, 1159

Beck, C., Schmidt, W., Rezaei, R., \& Rammacher, W. 2008, A\&A, 479, 213

Beckers, J. M. \& Tallant, P. E. 1969, Sol. Phys., 7, 351

Bellot Rubio, L. R., Collados, M., Ruiz Cobo, B., \& Rodríguez Hidalgo, I. 2000, ApJ, 534, 989

Bloomfield, D. S., Lagg, A., \& Solanki, S. K. 2007, ApJ, 671, 1005

Bogdan, T. J. \& Judge, P. G. 2006, Royal Society of London Philosophical Transactions Series A, 364, 313

Cally, P. S. \& Bogdan, T. J. 1997, ApJ, 486, L67

Carlsson, M. \& Stein, R. F. 1997, ApJ, 481, 500

Centeno, R., Collados, M., \& Trujillo Bueno, J. 2006, ApJ, 640, 1153

Centeno, R., Collados, M., \& Trujillo Bueno, J. 2009, ApJ, 692, 1211

Centeno, R., Trujillo Bueno, J., Uitenbroek, H., \& Collados, M. 2008, ApJ, 677, 742

Collados, M., Lagg, A., Díaz Garcí A, J. J., et al. 2007, in Astronomical Society of the Pacific Conference Series, Vol. 368 The Physics of Chromospheric Plasmas, ed. P. Heinzel, I. Dorotovič, \& R. J. Rutten, 611

Duvall, T. L., D'Silva, S., Jefferies, S. M., Harvey, J. W., \& Schou, J. 1996, Nature, 379, 235

Felipe, T., Khomenko, E., \& Collados, M. 2010, ApJ, 719, 357

Filippenko, A. V. 1982, PASP, 94, 715
Giovanelli, R. G. 1972, Sol. Phys., 27, 71

Giovanelli, R. G., Harvey, J. W., \& Livingston, W. C. 1978, Sol. Phys., 58, 347

Gurman, J. B. \& Leibacher, J. W. 1984, ApJ, 283, 859

Hindman, B. W., Jain, R., \& Zweibel, E. G. 1997, ApJ, 476, 392

Khomenko, E. \& Collados, M. 2009, A\&A, 506, L5

Khomenko, E., Kosovichev, A., Collados, M., Parchevsky, K., \& Olshevsky, V. 2009, ApJ, 694, 411

Khomenko, E. V., Collados, M., \& Bellot Rubio, L. R. 2003, ApJ, 588,606

Kobanov, N. I. 1990, Sol. Phys., 125, 25

Kostyk, R. I. \& Khomenko, E. V. 2002, Astronomy Reports, 46, 925

Krijger, J. M., Rutten, R. J., Lites, B. W., et al. 2001, A\&A, 379, 1052

Lites, B. W. 1984, ApJ, 277, 874

Lites, B. W. 1986, ApJ, 301, 1005

Lites, B. W. 1992, in NATO ASIC Proc. 375: Sunspots. Theory and Observations, ed. J. H. Thomas \& N. O. Weiss, 261-302

Lites, B. W., Rutten, R. J., \& Kalkofen, W. 1993, ApJ, 414, 345

Lites, B. W., Thomas, J. H., Bogdan, T. J., \& Cally, P. S. 1998, ApJ, 497, 464

Liu, S. \& Smith, E. V. P. 1972, Sol. Phys., 24, 301

Maltby, P., Avrett, E. H., Carlsson, M., et al. 1986, ApJ, 306, 284

Rouppe van der Voort, L. H. M., Rutten, R. J., Sütterlin, P., Sloover, P. J., \& Krijger, J. M. 2003, å, 403, 277

Spiegel, E. A. 1957, ApJ, 126, 202

Thomas, J. H., Cram, L. E., \& Nye, A. H. 1982, Nature, 297, 485 von der Luehe, O., Soltau, D., Berkefeld, T., \& Schelenz, T. 2003, in Proceedings of the SPIE, Vol. 4853, Innovative Telescopes and Instrumentation for Solar Astrophysics, ed. S. L. Keil \& S. V. Avakyan, 187-193

Zirin, H. \& Stein, A. 1972, ApJ, 178, L85+ 